\title{
An Efficient Approach for Two-Dimensional Parameter Estimation of a Single-Tone
}

\author{
H. C. So, Frankie K. W. Chan, W. H. Lau, and Cheung-Fat Chan
}

\begin{abstract}
In this paper, parameter estimation of a two-dimensional (2-D) single damped real/complex tone in the presence of additive white Gaussian noise is addressed. By utilizing the rank-one property of the 2-D noise-free data matrix, the damping factor and frequency for each dimension are estimated in a separable manner from the principal left and right singular vectors according to an iterative weighted least squares procedure. The remaining parameters are then obtained straightforwardly using standard least squares. The biases as well as variances of the damping factor and frequency estimates are also derived, which show that they are approximately unbiased and their performance achieves Cramér-Rao lower bound (CRLB) at sufficiently large signal-to-noise ratio (SNR) and/or data size conditions. We refer the proposed approach to as principal-singular-vector utilization for modal analysis (PUMA) which performs estimation in a fast and accurate manner. The development and analysis can easily be adapted for a tone which is undamped in at least one dimension. Furthermore, comparative simulation results with several conventional 2-D estimators and CRLB are included to corroborate the theoretical development of the PUMA approach as well as to demonstrate its superiority.
\end{abstract}

Index Terms-Linear prediction, modal analysis, principal singular vectors, two-dimensional frequency estimation, weighted least squares.

\section{INTRODUCTION}

$\mathbf{P}$ ARAMETER estimation of two-dimensional (2-D) damped/undamped sinusoidal signals in noise has been an important research topic because of its numerous applications such as angle-of-arrival estimation with a 2-D sensor array [1], synthetic aperture radar imaging [2], frequency and wave-number estimation in array processing [3], nuclear magnetic resonance (NMR) spectroscopy [4], joint incidence angle and path delay estimation in wireless communications [5], and health assessment of living trees [6].

In the presence of white Gaussian noise, the maximum-likelihood (ML) estimator [7] can attain optimum performance but its computational load is extremely heavy due to the requirement of a multidimensional search. To avoid the demanding search, relaxation strategies for the ML approach

Manuscript received September 08, 2009; accepted November 16, 2009. First published December 18, 2009; current version published March 10, 2010. The associate editor coordinating the review of this manuscript and approving it for publication was Prof. Andreas Jakobsson.

The authors are with the Department of Electronic Engineering, City University of Hong Kong, Kowloon, Hong Kong (e-mail: ckwf@ hkexperts.com).

Color versions of one or more of the figures in this paper are available online at http://ieeexplore.ieee.org.

Digital Object Identifier 10.1109/TSP.2009.2038962 such as iterative quadratic maximum-likelihood (IQML) [7] and method of direction estimation (MODE) [1] schemes have been proposed where proper parameter initialization is needed for achieving near-optimum accuracy. Alternatively, subspace-based methodology is another popular choice for the 2-D sinusoidal parameter estimation problem, which includes multiple signal classification (MUSIC) [2], [4], estimation of signal parameters via rotational invariance techniques (ESPRIT) [5], [6], [8], and matrix pencil (MP) [9], [10] algorithms. Comparing with the ML estimator and its approximations, these methods are more computationally attractive at the expense of suboptimality. In the MUSIC methods, the main computation is to perform a 2-D peak search while in the ESPRIT and MP schemes, singular value decomposition (SVD) for a Hankel block-Hankel matrix whose size is larger than that of the original data matrix, corresponds to their most demanding procedure. In this paper, we contribute to the 2-D parameter estimation of a single damped/undamped real/complex tone. In short, the proposed approach, which is referred to as principal-singular-vector utilization for modal analysis (PUMA), is more computationally attractive than the subspace methods and its estimation performance achieves Cramér-Rao lower bound (CRLB) at sufficiently high signal-to-noise ratio (SNR) and/or large data size conditions. It is worthy to point out that 2-D single-tone parameter estimation has also received considerable attention but most of the related works [11], [12] consider the undamped cisoid only.

The rest of the paper is organized as follows. In Section II, we present the PUMA algorithm development for a damped complex tone. The key ideas are to make use of the rank-one property of the 2-D noise-free data matrix and find the damping factor as well as frequency parameters for each dimension from the principal left and right singular vectors in a separable manner. Based on linear prediction (LP) and weighted least squares (WLS), an iterative procedure that operates on the principal singular vectors is devised for the damping factor and frequency estimation, and this is analogous to the one-dimensional IQML method [13]-[15]. After the nonlinear parameters are determined, the remaining parameter, namely, complex amplitude, is then estimated straightforwardly using least squares (LS). Section III contributes to the estimation of a damped real tone. Interestingly, the rank of the corresponding noise-free data matrix is also equal to one, and we follow Section II to develop a similar estimation procedure. Mean and variance analysis of the proposed algorithms is provided in Section IV. Modifications for tackling a real/complex tone which is undamped in at least one dimension are discussed in Section V. Simulation results are included in Section VI to 
TABLE I

LIST OF SYMBOLS

\begin{tabular}{|c|c|}
\hline Symbol & Meaning \\
\hline$T$ & transpose \\
\hline${ }^{*}$ & conjugate transpose \\
\hline${ }^{-1}$ & complex conjugate \\
\hline$\dagger$ & pseudorse \\
\hline vec & vectorization \\
\hline$\otimes$ & Kronecker product \\
\hline $\mathbb{E}$ & expectation \\
\hline $\mathcal{O}$ & $i \times i$ identity matrix \\
\hline $\mathbf{I}_{i}$ & $i \times j$ zero matrix \\
\hline $\mathbf{0}_{i \times j}$ & noise-free value of $\mathbf{a}$ \\
\hline$\tilde{\mathbf{a}}$ & variable for $\mathbf{a}$ \\
\hline $\mathbf{\mathbf { a }}$ & perturbation of $\mathbf{a}$ \\
\hline$\Delta \mathbf{a}$ & $i$ th element of $\mathbf{a}$ \\
\hline$[\mathbf{a}]_{i}$ & $(i, j)$ entry of $\mathbf{A}$ \\
\hline$[\mathbf{A}]_{i, j}$ & estimate of $\mathbf{A}$ \\
\hline$\hat{\mathbf{A}}$ & trace of $\mathbf{A}$ \\
\hline $\operatorname{tr}(\mathbf{A})$ & rank of $\mathbf{A}$ \\
\hline $\operatorname{rank}(\mathbf{A})$ & diagonal matrix \\
\hline $\operatorname{diag}(\cdot)$ & Toeplitz matrix with first \\
\hline $\operatorname{Toeplitz}\left(\mathbf{a}, \mathbf{b}^{T}\right)$ & column a and first row $\mathbf{b}^{T}$ \\
\hline $\operatorname{var}(\cdot)$ & variance \\
\hline $\operatorname{cov}(\cdot)$ & covariance \\
\hline & \\
\hline & \\
\hline & \\
\hline & \\
\hline &
\end{tabular}

corroborate the analytical development and to evaluate the performance of the PUMA approach by comparing with the IQML [7] and ESPRIT algorithms [6], [8] as well as CRLB. Finally, conclusions are drawn in Section VI. A list of mathematical symbols that are used in the paper is given in Table I.

\section{ESTIMATION FOR DAMPED COMPLEX TONE}

In this section, parameter estimation of a single damped cisoid is addressed. The observed 2-D data model is

$r_{m, n}=s_{m, n}+q_{m, n}, \quad m=1,2, \cdots, M, \quad n=1,2, \cdots, N$

where

$$
s_{m, n}=\gamma \alpha^{m} \beta^{n} \exp \{j(\mu m+\nu n)\}
$$

is the noise-free signal. The $\gamma$ is the complex amplitude, $\mu \in$ $(-\pi, \pi)$ and $\nu \in(-\pi, \pi)$ are the frequencies while $\alpha>0$ and $\beta>0$ are their associated damping factors, and they are all unknown constants. The $q_{m, n}$ is a zero-mean complex white Gaussian process, that is, its real and imaginary components are real white processes with identical but unknown variances of $\sigma^{2} / 2$ and uncorrelated with each other. Without loss of generality, it is assumed that $M \geq N$. Given the $M N$ samples of $\left\{r_{m, n}\right\}$, our task is to find the cisoidal parameters, namely, $\mu$, $\nu, \alpha, \beta$, and $\gamma$.

To facilitate the algorithm development, we express (1) in matrix form as

$$
\mathbf{R}=\mathbf{S}+\mathbf{Q}
$$

where $\mathbf{R} \in \mathbb{C}^{M \times N}, \mathbf{S} \in \mathbb{C}^{M \times N}$, and $\mathbf{Q} \in \mathbb{C}^{M \times N}$ with $[\mathbf{R}]_{m, n}=r_{m, n},[\mathbf{S}]_{m, n}=s_{m, n}$, and $[\mathbf{Q}]_{m, n}=q_{m, n}$.

From the regular structure of $\mathbf{S}$, it is straightforward to see that the noise-free data matrix can be represented as

$$
\mathbf{S}=\gamma \mathbf{g h} \mathbf{h}^{T}
$$

where

$$
\mathbf{g}=\left[\begin{array}{llll}
\alpha \exp (j \mu) & (\alpha \exp (j \mu))^{2} & \cdots & (\alpha \exp (j \mu))^{M}
\end{array}\right]^{T}
$$

and

$$
\mathbf{h}=\left[\begin{array}{llll}
\beta \exp (j \nu) & (\beta \exp (j \nu))^{2} & \cdots & (\beta \exp (j \nu))^{N}
\end{array}\right]^{T}
$$

are complex vectors which are characterized by $\alpha$ and $\mu$, and $\beta$ and $\nu$, respectively. It is also observed that the elements in $\mathbf{g}$ and $\mathbf{h}$ satisfy the LP property:

$$
[\mathrm{g}]_{m}=a[\mathbf{g}]_{m-1}, \quad m=2,3, \cdots, M
$$

and

$$
[\mathbf{h}]_{n}=b[\mathbf{h}]_{n-1}, \quad n=2,3, \cdots, N
$$

where

$$
a=\alpha \exp (j \mu)
$$

and

$$
b=\beta \exp (j \nu) .
$$

On the other hand, $\mathbf{S}$ can be decomposed using SVD as

$$
\mathbf{S}=\tilde{\mathbf{U}} \tilde{\mathbf{\Lambda}} \tilde{\mathbf{V}}^{H}
$$

where $\tilde{\Lambda}=\operatorname{diag}\left(\tilde{\lambda}_{1}, \tilde{\lambda}_{2}, \ldots, \tilde{\lambda}_{N}\right)$ is the diagonal matrix of singular values of $\mathbf{S}$ with $\tilde{\lambda}_{1}>\tilde{\lambda}_{2}=\cdots=\tilde{\lambda}_{N}=0$ while $\tilde{\mathbf{U}}=$

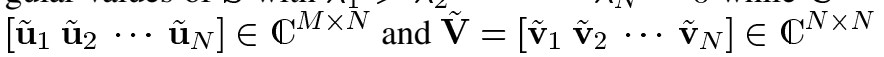
are orthonormal matrices whose columns are the corresponding left and right singular vectors, respectively. From the decomposition in (4)-(6), it is obvious that $\operatorname{rank}(\mathbf{S})=1$ and thus (11) can be simplified to

$$
\mathbf{S}=\tilde{\lambda}_{1} \tilde{\mathbf{u}}_{1} \tilde{\mathbf{v}}_{1}^{H} .
$$

That is, $\tilde{\lambda}_{1}, \tilde{\mathbf{u}}_{1}$, and $\tilde{\mathbf{v}}_{1}^{*}$, correspond to $\gamma, \mathbf{g}$, and $\mathbf{h}$, up to an unknown multiplying constant, respectively. Mathematically, we can write

$$
\mathrm{g}=k_{g} \tilde{\mathbf{u}}_{1}
$$

and

$$
\mathbf{h}^{*}=k_{h} \tilde{\mathbf{v}}_{1}
$$

where $k_{g}$ and $k_{h}$ are unknown complex constants. As $\tilde{\mathbf{u}}_{1}^{H} \tilde{\mathbf{u}}_{1}=\tilde{\mathbf{v}}_{1}^{H} \tilde{\mathbf{v}}_{1}=1$, we easily get $\mathbf{g}^{H} \mathbf{g}=\left|k_{g}\right|^{2}$ and $\mathbf{h}^{T} \mathbf{h}^{*}=\mathbf{h}^{H} \mathbf{h}=\left|k_{h}\right|^{2}$, implying that $k_{g}=|\mathrm{g}| \exp \left(j \varphi_{g}\right)$ and 
$k_{h}=|\mathbf{h}| \exp \left(-j \varphi_{h}\right)$ where $\varphi_{g}$ and $\varphi_{h}$ are unknowns. Hence, (13) and (14) can be expressed as

$$
\mathbf{g}=|\mathrm{g}| \exp \left(j \varphi_{g}\right) \tilde{\mathbf{u}}_{1}
$$

and

$$
\mathbf{h}=|\mathbf{h}| \exp \left(j \varphi_{h}\right) \tilde{\mathbf{v}}_{1}^{*} .
$$

Substituting (15) and (16) into (4) and equating the resultant expression with (12), we have

$$
\tilde{\lambda}_{1}=\gamma|\mathbf{g}||\mathbf{h}| \exp \left(j \varphi_{g}\right) \exp \left(j \varphi_{h}\right)
$$

Nevertheless, $\tilde{\mathbf{u}}_{1}$ and $\tilde{\mathbf{v}}_{1}$ possess the same LP property as in (7)-(10). Based on these findings, our strategy is first to estimate $\alpha$ and $\mu$, and $\beta$ and $\nu$ from $\tilde{\mathbf{u}}_{1}$ and $\tilde{\mathbf{v}}_{1}$, in a separable manner, and their estimates are then employed for finding $\gamma$. As only the principal singular vectors are utilized for the modal signal parameter estimation, we refer this technique to as PUMA.

By decomposing $\mathbf{R}$ as $\mathbf{R}=\mathbf{U} \boldsymbol{\Lambda} \mathbf{V}^{H}$, the best rank-one approximation or LS estimate of $\mathbf{S}$ is

$$
\hat{\mathbf{S}}=\lambda_{1} \mathbf{u}_{1} \mathbf{v}_{1}^{H}
$$

where $\lambda_{1}, \mathbf{u}_{1}$, and $\mathbf{v}_{1}$ are the noisy versions of $\tilde{\lambda}_{1}, \tilde{\mathbf{u}}_{1}$, and $\tilde{\mathbf{v}}_{1}$, respectively. Define

$$
\mathbf{x}_{1}=\left[\begin{array}{llll}
{\left[\mathbf{u}_{1}\right]_{1}} & {\left[\mathbf{u}_{1}\right]_{2}} & \cdots & {\left[\mathbf{u}_{1}\right]_{M-1}}
\end{array}\right]^{T}
$$

and

$$
\mathbf{x}_{2}=\left[\begin{array}{llll}
{\left[\mathbf{u}_{1}\right]_{2}} & {\left[\mathbf{u}_{1}\right]_{3}} & \cdots & {\left[\mathbf{u}_{1}\right]_{M}}
\end{array}\right]^{T} .
$$

From (7), we have

$$
\mathbf{x}_{1} a \approx \mathbf{x}_{2} .
$$

Following [15], the WLS estimate of $a$ is computed as

$$
\hat{a}=\arg \min _{\breve{a}}\left(\mathbf{x}_{1} \check{a}-\mathbf{x}_{2}\right)^{H} \mathbf{\Psi}(a)\left(\mathbf{x}_{1} \check{a}-\mathbf{x}_{2}\right)=\frac{\mathbf{x}_{1}^{H} \mathbf{\Psi}(a) \mathbf{x}_{2}}{\mathbf{x}_{1}^{H} \mathbf{\Psi}(a) \mathbf{x}_{1}}
$$

where the optimum weighting matrix $\Psi(a)$ is constructed from the residual error of $\left(\mathbf{x}_{1} a-\mathbf{x}_{2}\right)$ and hence a function of $a$, which is commonly known as the Markov estimate [16], [17]. With the use of (19), (20), the inverse of $\boldsymbol{\Psi}(a)$ is

$$
\begin{aligned}
\boldsymbol{\Psi}^{-1}(a) & =\mathbb{E}\left\{\left(\mathbf{x}_{1} a-\mathbf{x}_{2}\right)\left(\mathbf{x}_{1} a-\mathbf{x}_{2}\right)^{H}\right\} \\
& =\mathbf{A} \mathbb{E}\left\{\mathbf{u}_{1} \mathbf{u}_{1}^{H}\right\} \mathbf{A}^{H}
\end{aligned}
$$

where

$$
\mathbf{A}=\operatorname{Toeplitz}\left(\left[\begin{array}{ll}
-a & \mathbf{0}_{1 \times(M-2)}
\end{array}\right]^{T},\left[\begin{array}{lll}
-a & 1 & \mathbf{0}_{1 \times(M-2)}
\end{array}\right]\right) \text {. }
$$

Let $\mathbf{u}_{1}=\tilde{\mathbf{u}}_{1}+\Delta \mathbf{u}_{1}$ where $\Delta \mathbf{u}_{1}$ is the perturbation of $\mathbf{u}_{1}$. At the ideal value of $a, \tilde{\mathbf{x}}_{1} a=\tilde{\mathbf{x}}_{2}$ or $\mathbf{A} \tilde{\mathbf{u}}_{1}=\mathbf{0}_{(M-1) \times 1}$ and thus (23) is equivalent to

$$
\Psi^{-1}(a)=\mathbf{A} \mathbb{E}\left\{\Delta \mathbf{u}_{1} \Delta \mathbf{u}_{1}^{H}\right\} \mathbf{A}^{H} .
$$

To compute (24), we first utilize [18] to obtain:

$$
\begin{aligned}
\Delta \mathbf{u}_{1} & \approx \tilde{\lambda}_{1}^{-1} \tilde{\mathbf{U}}_{n} \tilde{\mathbf{U}}_{n}^{H} \mathbf{Q} \tilde{\mathbf{v}}_{1} \\
& =\tilde{\lambda}_{1}^{-1}\left(\tilde{\mathbf{v}}_{1}^{T} \otimes \tilde{\mathbf{U}}_{n} \tilde{\mathbf{U}}_{n}^{H}\right) \operatorname{vec}(\mathbf{Q})
\end{aligned}
$$

where $\tilde{\mathbf{U}}_{n}=\left[\begin{array}{llll}\tilde{\mathbf{u}}_{2} & \tilde{\mathbf{u}}_{3} & \cdots & \tilde{\mathbf{u}}_{N}\end{array}\right]$ is the noise-free version of $\mathbf{U}_{n}=$ $\left[\begin{array}{llll}\mathbf{u}_{2} & \mathbf{u}_{3} & \cdots & \mathbf{u}_{N}\end{array}\right]$, which corresponds to the noise subspace. With the use of (25), $\mathbb{E}\left\{\boldsymbol{\Delta} \mathbf{u}_{1} \Delta \mathbf{u}_{1}^{H}\right\}$ is derived as

$$
\begin{aligned}
\mathbb{E}\{ & \left.\Delta \mathbf{u}_{1} \Delta \mathbf{u}_{1}^{H}\right\} \\
= & \tilde{\lambda}_{1}^{-2}\left(\tilde{\mathbf{v}}_{1}^{T} \otimes \tilde{\mathbf{U}}_{n} \tilde{\mathbf{U}}_{n}^{H}\right) \mathbb{E}\left\{\operatorname{vec}(\mathbf{Q}) \operatorname{vec}(\mathbf{Q})^{H}\right\} \\
& \times\left(\tilde{\mathbf{v}}_{1}^{*} \otimes \tilde{\mathbf{U}}_{n} \tilde{\mathbf{U}}_{n}^{H}\right) \\
= & \tilde{\lambda}_{1}^{-2}\left(\tilde{\mathbf{v}}_{1}^{T} \otimes \tilde{\mathbf{U}}_{n} \tilde{\mathbf{U}}_{n}^{H}\right)\left(\sigma^{2} \mathbf{I}_{M N}\right)\left(\tilde{\mathbf{v}}_{1}^{*} \otimes \tilde{\mathbf{U}}_{n} \tilde{\mathbf{U}}_{n}^{H}\right) \\
= & \tilde{\lambda}_{1}^{-2} \sigma^{2}\left(\tilde{\mathbf{v}}_{1}^{T} \tilde{\mathbf{v}}_{1}^{*}\right) \otimes\left(\tilde{\mathbf{U}}_{n} \tilde{\mathbf{U}}_{n}^{H} \tilde{\mathbf{U}}_{n} \tilde{\mathbf{U}}_{n}^{H}\right) \\
= & \tilde{\lambda}_{1}^{-2} \sigma^{2} \tilde{\mathbf{U}}_{n} \tilde{\mathbf{U}}_{n}^{H} .
\end{aligned}
$$

Note that the values of $\tilde{\lambda}_{1}$ and $\sigma^{2}$ are not required as they will be canceled out in (22), that is, we only need to know $\Psi(a)$ up to a multiplying scalar. Employing $\mathbf{A} \tilde{\mathbf{u}}_{1}=\mathbf{0}_{(M-1) \times 1}$ and $\tilde{\mathbf{U}}_{n} \tilde{\mathbf{U}}_{n}^{H}=\mathbf{I}_{M}-\tilde{\mathbf{u}}_{1} \tilde{\mathbf{u}}_{1}^{H}$, the weighting matrix of (24) is simplified as

$$
\Psi(a)=\left(\mathbf{A} \tilde{\mathbf{U}}_{n} \tilde{\mathbf{U}}_{n}^{H} \mathbf{A}^{H}\right)^{-1}=\left(\mathbf{A} \mathbf{A}^{H}\right)^{-1} .
$$

As $\boldsymbol{\Psi}(a)$ is characterized by the unknown parameter $a$, we follow [15] to estimate $\mu$ and $\alpha$ in an iterative manner and the estimation procedure is summarized as follows.

i) Set $\boldsymbol{\Psi}(a)=\mathbf{I}_{M-1}$.

ii) Calculate $\hat{a}$ using (22)

iii) Compute an updated version of $\Psi(a)$ using (27) with $a=$ $\hat{a}$.

iv) Repeat Steps ii)-iii) until a stopping criterion is reached.

v) The damping factor and frequency estimates are estimated from the magnitude and phase of $\hat{a}$ according to

$$
\hat{\alpha}=|\hat{a}|
$$

and

$$
\hat{\mu}=\angle(\hat{a}) .
$$

Applying the same idea in $\mathbf{v}_{1}$, we have

$$
\mathbf{y}_{1} b^{*} \approx \mathbf{y}_{2}
$$

where $\mathbf{y}_{1}=\left[\begin{array}{llll}{\left[\mathbf{v}_{1}\right]_{1}} & {\left[\mathbf{v}_{1}\right]_{2}} & \cdots & {\left[\mathbf{v}_{1}\right]_{N-1}}\end{array}\right]^{T}$ and $\mathbf{y}_{2}=$ $\left[\begin{array}{llll}{\left[\mathbf{v}_{1}\right]_{2}} & {\left[\mathbf{v}_{1}\right]_{3}} & \cdots & {\left[\mathbf{v}_{1}\right]_{N}}\end{array}\right]^{T}$. Following the development in (22)-(27), the conceptual solution for $b$ is

$$
\hat{b}=\left(\frac{\mathbf{y}_{1}^{H} \mathbf{\Upsilon}(b) \mathbf{y}_{2}}{\mathbf{y}_{1}^{H} \mathbf{\Upsilon}(b) \mathbf{y}_{1}}\right)^{*}
$$

where

$$
\mathbf{\Upsilon}(b)=\left(\mathbf{B B}^{H}\right)^{-1}
$$

with

$$
\mathbf{B}=\operatorname{Toeplitz}\left(\left[\begin{array}{ll}
-b^{*} & \mathbf{0}_{1 \times(N-2)}
\end{array}\right]^{T},\left[\begin{array}{lll}
-b^{*} & 1 & \mathbf{0}_{1 \times(N-2)}
\end{array}\right]\right) .
$$

In practice, $\hat{\nu}$ and $\hat{\beta}$ are obtained using the iterative procedure which is analogous to estimating $\mu$ and $\alpha$. 
Employing the damping factor and frequency estimates, we construct $\hat{\mathrm{g}}=\left[\hat{\alpha} \exp (j \hat{\mu})(\hat{\alpha} \exp (j \hat{\mu}))^{2} \cdots(\hat{\alpha} \exp (j \hat{\mu}))^{M}\right]^{T}$ and $\hat{\mathbf{h}}=\left[\hat{\beta} \exp (j \hat{\nu})(\hat{\beta} \exp (j \hat{\nu}))^{2} \cdots(\hat{\beta} \exp (j \hat{\nu}))^{N}\right]^{T}$ to estimate $\gamma$ as follows. From (4), we have

$$
\mathbf{R} \approx \gamma \hat{\mathbf{g}} \hat{\mathbf{h}}^{T}
$$

Vectorizing both sides of (33) yields

$$
\operatorname{vec}(\mathbf{R}) \approx \gamma \hat{\mathbf{h}} \otimes \hat{\mathrm{g}} .
$$

The LS estimate of $\gamma$ is straightforwardly obtained as

$$
\hat{\gamma}=(\hat{\mathbf{h}} \otimes \hat{\mathbf{g}})^{\dagger} \operatorname{vec}(\mathbf{R}) .
$$

\section{ESTIMATION FOR DAMPED REAL TONE}

For parameter estimation of a damped real tone, the signal model is

$r_{m, n}=s_{m, n}+q_{m, n}, \quad m=1,2, \cdots, M, \quad n=1,2, \cdots, N$

where

$$
s_{m, n}=\gamma \alpha^{m} \beta^{n} \cos (\mu m+\phi) \cos (\nu n+\theta)
$$

is the noise-free sinusoid. Now $\gamma>0$ is the real-valued amplitude, $\mu \in(0, \pi), \alpha>0$ and $\phi \in[0,2 \pi)$ are the frequency, damping factor and phase for one dimension while $\nu \in(0, \pi)$, $\beta>0$ and $\theta \in[0,2 \pi)$ are the corresponding parameters for another dimension. The $q_{m, n}$ is assumed to be a real zero-mean white Gaussian process with unknown variance $\sigma^{2}$. Expressing (37) in matrix form, we find that it can be decomposed as in (4) but $\mathbf{g}$ and $\mathbf{h}$ are modified to

$$
\mathbf{g}=\left[\begin{array}{ll}
\alpha \cos (\mu+\phi) & \alpha^{2} \cos (2 \mu+\phi) \alpha^{M} \cos (M \mu+\phi)^{T}
\end{array}\right]
$$

and

$$
\mathbf{h}=\left[\beta \cos (\nu+\theta) \beta^{2} \cos (2 \nu+\theta) \cdots \beta^{N} \cos (N \nu+\theta)^{T}\right] .
$$

The LP property in $\mathbf{g}$ and $\mathbf{h}$ can easily be observed as

$$
[\mathbf{g}]_{m}=a_{1}[\mathbf{g}]_{m-1}+a_{2}[\mathbf{g}]_{m-2}, \quad m=3,4, \cdots, M
$$

and

$$
[\mathbf{h}]_{n}=b_{1}[\mathbf{h}]_{n-1}+b_{2}[\mathbf{h}]_{n-2}, \quad n=3,4, \cdots, N
$$

where

$$
\begin{aligned}
& a_{1}=2 \alpha \cos (\mu) \\
& a_{2}=-\alpha^{2} \\
& b_{1}=2 \beta \cos (\nu)
\end{aligned}
$$

and

$$
b_{2}=-\beta^{2}
$$

Similar to (12)-(17), we can also express $\mathbf{S}$ according to SVD as

$$
\mathbf{S}=\tilde{\lambda}_{1} \tilde{\mathbf{u}}_{1} \tilde{\mathbf{v}}_{1}^{T}
$$

where $\mathbf{g}=|\mathbf{g}| \tilde{\mathbf{u}}_{1}$, and $\tilde{\lambda}_{1}=\gamma|\mathbf{g}||\mathbf{h}|$. By decomposing $\mathbf{R}$ as $\mathbf{R}=\mathbf{U} \boldsymbol{\Lambda} \mathbf{V}^{T}$ and utilizing the LP property of (40) in matrix form, we have

$$
\mathrm{Xa} \approx \mathrm{x}
$$

where

$$
\begin{aligned}
& \mathbf{X}=\text { Toeplitz }\left(\left[\left[\mathbf{u}_{1}\right]_{2}\left[\mathbf{u}_{1}\right]_{3} \cdots\left[\mathbf{u}_{1}\right]_{M-1}\right]^{T},\left[\left[\mathbf{u}_{1}\right]_{2}\left[\mathbf{u}_{1}\right]_{1}\right]\right) \\
& \mathbf{x}=\left[\left[\mathbf{u}_{1}\right]_{3}\left[\mathbf{u}_{1}\right]_{4} \cdots\left[\mathbf{u}_{1}\right]_{M}\right]^{T}
\end{aligned}
$$

and

$$
\mathbf{a}=\left[\begin{array}{ll}
a_{1} & a_{2}
\end{array}\right]^{T}
$$

Following the development in (22)-(27), the WLS estimate of $\mathbf{a}$ is

$$
\hat{\mathbf{a}}=\left(\mathbf{X}^{T} \Psi(\mathbf{a}) \mathbf{X}\right)^{-1}\left(\mathbf{X}^{T} \Psi(\mathbf{a}) \mathbf{x}\right)
$$

where

$$
\Psi(\mathbf{a})=\left(\mathbf{A A}^{T}\right)^{-1}
$$

with

$$
\begin{array}{r}
\mathbf{A}=\operatorname{Toeplitz}\left(\left[\begin{array}{ll}
-a_{2} & \mathbf{0}_{1 \times(M-3)}
\end{array}\right]^{T},\right. \\
\left.\left[\begin{array}{llll}
-a_{2} & -a_{1} & 1 & \mathbf{0}_{1 \times(M-3)}
\end{array}\right]\right) .
\end{array}
$$

As $\Psi(\mathbf{a})$ is a function of $\mathbf{a}$, we first set $\Psi(\mathbf{a})=\mathbf{I}_{M-2}$ and iterate between (51) and (52) as in Section II to calculate a. Once $\hat{\mathbf{a}}$ is available, the damping factor and frequency are estimated using (42) and (43):

$$
\hat{\alpha}=\sqrt{-\hat{a}_{2}}
$$

and

$$
\hat{\mu}=\cos ^{-1}\left(\frac{\hat{a}_{1}}{2 \hat{\alpha}}\right) .
$$

To estimate $\phi$, we consider

$$
\mathbf{R} \approx \gamma \mathbf{g} \mathbf{h}^{T} \approx \gamma \mathbf{G} \varrho \mathbf{h}^{T}
$$

where

$$
\mathbf{G}=\left[\begin{array}{cccc}
\hat{\alpha} \cos (\hat{\mu}) & \hat{\alpha}^{2} \cos (2 \hat{\mu}) & \cdots & \hat{\alpha}^{M} \cos (M \hat{\mu}) \\
-\hat{\alpha} \sin (\hat{\mu}) & -\hat{\alpha}^{2} \sin (2 \hat{\mu}) & \cdots & -\hat{\alpha}^{M} \sin (M \hat{\mu})
\end{array}\right]^{T}
$$

and

$$
\varrho=\left[\begin{array}{ll}
\cos (\phi) & \sin (\phi)
\end{array}\right]^{T} .
$$


The former is constructed from $\hat{\mu}$ and $\hat{\alpha}$ while the latter is a function of $\phi$. Pre-multiplying both sides of (55) by $\mathbf{G}^{\dagger}$ yields

$$
\mathbf{G}^{\dagger} \mathbf{R} \approx \gamma \varrho \mathbf{h}^{T}=\left[\begin{array}{ll}
\gamma \cos (\phi) \mathbf{h} & \gamma \sin (\phi) \mathbf{h}
\end{array}\right]^{T} .
$$

It follows from (58) that

$$
\boldsymbol{g}_{1} \tan (\phi) \approx \boldsymbol{g}_{2}
$$

where

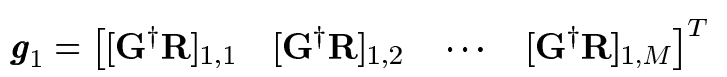

and

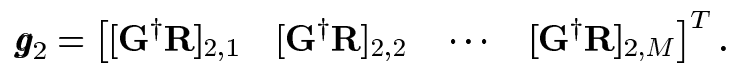

Based on (59), the LS estimate of $\phi$ is

$$
\hat{\phi}=\tan ^{-1}\left(\boldsymbol{g}_{1}^{\dagger} \boldsymbol{g}_{2}\right) \text {. }
$$

Likewise, the conceptual solution for $\mathbf{b}=\left[\begin{array}{ll}b_{1} & b_{2}\end{array}\right]^{T}$ is

$$
\hat{\mathbf{b}}=\left(\mathbf{Y}^{T} \boldsymbol{\Upsilon}(\mathbf{b}) \mathbf{Y}\right)^{-1} \mathbf{Y}^{T} \boldsymbol{\Upsilon}(\mathbf{b}) \mathbf{y}
$$

where

$$
\begin{aligned}
& \Upsilon \mathbf{( b )}=\left(\mathbf{B B}^{T}\right)^{-1} \\
& \mathbf{B}=\operatorname{Toeplitz}\left(\left[\begin{array}{ll}
-b_{2} & \mathbf{0}_{1 \times(N-3)}
\end{array}\right]^{T},\right. \\
& \left.\left[\begin{array}{llll}
-b_{2} & -b_{1} & 1 & \mathbf{0}_{1 \times(N-3)}
\end{array}\right]\right)
\end{aligned}
$$

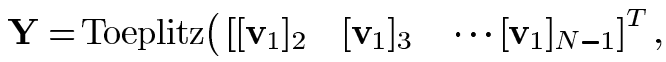

$$
\begin{aligned}
& \left.\left[\left[\mathbf{v}_{1}\right]_{2} \quad\left[\mathbf{v}_{1}\right]_{1}\right]\right)
\end{aligned}
$$

and

$$
\mathbf{y}=\left[\begin{array}{llll}
{\left[\mathbf{v}_{1}\right]_{3}} & {\left[\mathbf{v}_{1}\right]_{4}} & \cdots & {\left[\mathbf{v}_{1}\right]_{N}}
\end{array}\right]^{T} .
$$

After finding $\hat{\nu}$ and $\hat{\beta}$ using the same iterative procedure, we follow the development in (55)-(62) to obtain the LS estimate of $\theta$ :

$$
\hat{\theta}=\tan ^{-1}\left(\boldsymbol{h}_{1}^{\dagger} \boldsymbol{h}_{2}\right)
$$

where

$$
\begin{aligned}
& h_{1}=\left[\left[\mathbf{R}\left(\mathbf{H}^{T}\right)^{\dagger}\right]_{1,1}\left[\mathbf{R}\left(\mathbf{H}^{T}\right)^{\dagger}\right]_{2,1} \cdots\left[\mathbf{R}\left(\mathbf{H}^{T}\right)^{\dagger}\right]_{N, 1}\right]^{T} \\
& h_{2}=\left[\left[\mathbf{R}\left(\mathbf{H}^{T}\right)^{\dagger}\right]_{1,2}\left[\mathbf{R}\left(\mathbf{H}^{T}\right)^{\dagger}\right]_{2,2} \cdots\left[\mathbf{R}\left(\mathbf{H}^{T}\right)^{\dagger}\right]_{N, 2}\right]^{T}
\end{aligned}
$$

and

$$
\mathbf{H}=\left[\begin{array}{cccc}
\hat{\beta} \cos (\hat{\nu}) & \hat{\beta}^{2} \cos (2 \hat{\nu}) & \cdots & \hat{\beta}^{N} \cos (N \hat{\nu}) \\
-\hat{\beta} \sin (\hat{\nu}) & -\hat{\beta}^{2} \sin (2 \hat{\nu}) & \cdots & -\hat{\beta}^{N} \sin (N \hat{\nu})
\end{array}\right]^{T} .
$$

Analogous to Section II, the LS estimate of $\gamma$ is

$$
\hat{\gamma}=(\hat{\mathbf{h}} \otimes \hat{\mathbf{g}})^{\dagger} \operatorname{vec}(\mathbf{R})
$$

where

$$
\hat{\mathrm{g}}=\left[\begin{array}{llll}
\hat{\alpha} \cos (\hat{\mu}+\hat{\phi}) & \hat{\alpha}^{2} \cos (2 \hat{\mu}+\hat{\phi}) & \cdots & \hat{\alpha}^{M} \cos (M \hat{\mu}+\hat{\phi})
\end{array}\right]^{T}
$$
and

$$
\hat{\mathbf{h}}=\left[\begin{array}{llll}
\hat{\beta} \cos (\hat{\nu}+\hat{\theta}) & \hat{\beta}^{2} \cos (2 \hat{\nu}+\hat{\theta}) & \cdots & \hat{\beta}^{N} \cos (N \hat{\nu}+\hat{\theta})
\end{array}\right]^{T} .
$$

\section{Performance Analysis}

In this section, the biases and variances of the damping factor and frequency estimates are analyzed. The complex and real data models are investigated one by one as follows.

\section{A. Complex Tone}

The basic idea for our analysis is to utilize (22). Upon convergence of the iterative procedure, the estimate of $a$ should satisfy

$$
\hat{a}=\frac{\mathbf{x}_{1}^{H} \mathbf{\Psi}(\hat{a}) \mathbf{x}_{2}}{\mathbf{x}_{1}^{H} \Psi(\hat{a}) \mathbf{x}_{1}} .
$$

Based on (73), we construct a function $f(\breve{a})$ :

$$
\begin{aligned}
f(\check{a}) & =\mathbf{x}_{1}^{H} \boldsymbol{\Psi}(\check{a}) \mathbf{x}_{1} \check{a}-\mathbf{x}_{1}^{H} \boldsymbol{\Psi}(\check{a}) \mathbf{x}_{2} \\
& =\mathbf{x}_{1}^{H} \boldsymbol{\Psi}(\check{a})\left(\mathbf{x}_{1} \check{a}-\mathbf{x}_{2}\right)
\end{aligned}
$$

such that $f(\hat{a})=0$. For sufficiently large SNR and/or data size, $\hat{a}$ will be located at a reasonable proximity of $a$. Using Taylor's series to expand $f(\hat{a})$ around $a$ up to the first-order term, we get

$$
0=f(\hat{a}) \approx f(a)+f^{\prime}(a)(\hat{a}-a)
$$

where $f^{\prime}(a)$ is the first derivative of $f(\check{a})$ evaluated at $\breve{a}=a$. Expressing $\mathbf{x}_{1}=\tilde{\mathbf{x}}_{1}+\Delta \mathbf{x}_{1}$ and $\mathbf{x}_{2}=\tilde{\mathbf{x}}_{2}+\Delta \mathbf{x}_{2}$ as well as using $\tilde{\mathbf{x}}_{1} a=\tilde{\mathbf{x}}_{2}, f(a)$ can be linearized as

$$
\begin{aligned}
f(a) & =\left(\tilde{\mathbf{x}}_{1}+\boldsymbol{\Delta} \mathbf{x}_{1}\right)^{H} \boldsymbol{\Psi}(a)\left[\left(\tilde{\mathbf{x}}_{1}+\boldsymbol{\Delta} \mathbf{x}_{1}\right) a-\left(\tilde{\mathbf{x}}_{2}+\boldsymbol{\Delta} \mathbf{x}_{2}\right)\right] \\
& \approx \tilde{\mathbf{x}}_{1}^{H} \Psi(a)\left(\boldsymbol{\Delta} \mathbf{x}_{1} a-\boldsymbol{\Delta} \mathbf{x}_{2}\right) \\
& \approx \tilde{\mathbf{x}}_{1}^{H} \Psi(a) \mathbf{A} \boldsymbol{\Delta} \mathbf{u}_{1} .
\end{aligned}
$$

On the other hand, $f^{\prime}(a)$ is approximated as

$$
\begin{aligned}
f^{\prime}(a) & =\mathbf{x}_{1}^{H} \Psi(a) \mathbf{x}_{1}-\mathbf{x}_{1}^{H} \Psi(a)\left(\Psi^{-1}(a)\right)^{\prime} \Psi(a)\left(\mathbf{x}_{1} a-\mathbf{x}_{2}\right) \\
& \approx \tilde{\mathbf{x}}_{1}^{H} \Psi(a) \tilde{\mathbf{x}}_{1} .
\end{aligned}
$$

Combining (75)-(77), we have

$$
\begin{aligned}
\hat{a} & \approx a-\frac{f(a)}{f^{\prime}(a)} \\
& \approx a-\frac{\tilde{\mathbf{x}}_{1}^{H} \mathbf{\Psi}(a) \mathbf{A} \mathbf{\Delta} \mathbf{u}_{1}}{\tilde{\mathbf{x}}_{1}^{H} \mathbf{\Psi}(a) \tilde{\mathbf{x}}_{1}}
\end{aligned}
$$


As $\tilde{\mathbf{x}}_{1}^{H} \mathbf{\Psi}(a) \tilde{\mathbf{x}}_{1}$ is deterministic and $\mathbb{E}\left\{\Delta \mathbf{u}_{1}\right\}=\mathbf{0}_{(M-1) \times 1}$, it is clear that $\mathbb{E}\{\hat{a}\} \approx a$ and hence $\hat{a}$ is approximately unbiased for sufficiently large SNR and data size conditions. Employing (24) and (78), the mean-square error or variance of $\hat{a}$ is derived as

$$
\begin{aligned}
\operatorname{var}(\hat{a}) & =\mathbb{E}\left\{(\hat{a}-a)(\hat{a}-a)^{*}\right\} \\
& \approx \frac{\tilde{\mathbf{x}}_{1}^{H} \mathbf{\Psi}(a) \mathbf{A} \mathbb{E}\left\{\boldsymbol{\Delta} \mathbf{u}_{1} \Delta \mathbf{u}_{1}^{H}\right\} \mathbf{A}^{H} \mathbf{\Psi}(a) \tilde{\mathbf{x}}_{1}}{\left(\tilde{\mathbf{x}}_{1}^{H} \mathbf{\Psi}(a) \tilde{\mathbf{x}}_{1}\right)^{2}} \\
& =\frac{\tilde{\mathbf{x}}_{1}^{H} \boldsymbol{\Psi}(a)\left(\tilde{\lambda}_{1}^{-2} \sigma^{2} \boldsymbol{\Psi}^{-1}(a)\right) \boldsymbol{\Psi}(a) \tilde{\mathbf{x}}_{1}}{\left(\tilde{\mathbf{x}}_{1}^{H} \mathbf{\Psi}(a) \tilde{\mathbf{x}}_{1}\right)^{2}} \\
& =\frac{\sigma^{2}}{\tilde{\lambda}_{1}^{2} \tilde{\mathbf{x}}_{1}^{H} \boldsymbol{\Psi}(a) \tilde{\mathbf{x}}_{1}} .
\end{aligned}
$$

Based on (79) and [19], the variances of $\hat{\mu}$ and $\hat{\alpha}$ are computed as

$$
\operatorname{var}(\hat{\mu}) \approx \frac{\operatorname{var}(\hat{a})}{2|a|^{2}} \approx \frac{\sigma^{2}}{2 \tilde{\lambda}_{1}^{2} \alpha^{2} \tilde{\mathbf{x}}_{1}^{H} \Psi(a) \tilde{\mathbf{x}}_{1}}
$$

and

$$
\operatorname{var}(\hat{\alpha}) \approx \frac{\alpha^{2} \operatorname{var}(\hat{a})}{2|a|^{2}} \approx \frac{\sigma^{2}}{2 \tilde{\lambda}_{1}^{2} \tilde{\mathbf{x}}_{1}^{H} \mathbf{\Psi}(a) \tilde{\mathbf{x}}_{1}} .
$$

From (80) and (81), we see that the expressions of $\operatorname{var}(\hat{\mu})$ and $\operatorname{var}(\hat{\alpha})$ are similar except that the former is inversely proportional to $\alpha^{2}$. Apart from this, both increase with the noise power and decrease with $\tilde{\lambda}_{1}^{2}$ and $\tilde{\mathbf{x}}_{1}^{H} \mathbf{\Psi}(a) \tilde{\mathbf{x}}_{1}$ which relates to the signal power and $M$.

Likewise, the variances of $\hat{\nu}$ and $\hat{\beta}$ are determined as

$$
\operatorname{var}(\hat{\nu}) \approx \frac{\sigma^{2}}{2 \tilde{\lambda}_{1}^{2} \beta^{2} \tilde{\mathbf{y}}_{1}^{H} \boldsymbol{\Upsilon}(b) \tilde{\mathbf{y}}_{1}}
$$

and

$$
\operatorname{var}(\hat{\beta}) \approx \frac{\sigma^{2}}{2 \tilde{\lambda}_{1}^{2} \tilde{\mathbf{y}}_{1}^{H} \mathbf{\Upsilon}(b) \tilde{\mathbf{y}}_{1}}
$$

\section{B. Real Tone}

Following the development in (73)-(79), it is shown that $\mathbb{E}\{\hat{\mathbf{a}}\} \approx \mathbf{a}$ and the covariance of $\hat{\mathbf{a}}$ in (51) is

$$
\operatorname{cov}(\hat{\mathbf{a}})=\mathbb{E}\left\{(\hat{\mathbf{a}}-\mathbf{a})(\hat{\mathbf{a}}-\mathbf{a})^{T}\right\} \approx \tilde{\lambda}_{1}^{-2} \sigma^{2}\left(\tilde{\mathbf{X}}^{T} \Psi(\mathbf{a}) \tilde{\mathbf{X}}\right)^{-1}
$$

Let $\boldsymbol{\rho}=[\mu \alpha]^{T}$. With the use of (42) and (43) and expanding $\hat{\mathbf{a}}$ around $\mathbf{a}$ with Taylor series up to the first-order term yields

$$
\hat{\mathbf{a}} \approx \mathbf{a}+\mathbf{C}(\hat{\boldsymbol{\rho}}-\rho)
$$

where

$$
\mathbf{C}=\left.\left[\begin{array}{ll}
\frac{\partial \check{a}_{1}}{\partial \mu} & \frac{\partial \check{a}_{1}}{\partial \check{\alpha}} \\
\frac{\partial \ddot{a}_{2}}{\partial \breve{\mu}} & \frac{\partial \check{a}_{2}}{\partial \check{\alpha}}
\end{array}\right]\right|_{\check{\mu}=\mu, \check{\alpha}=\alpha}=\left[\begin{array}{cc}
-2 \alpha \sin (\mu) & 2 \cos (\mu) \\
0 & -2 \alpha
\end{array}\right] .
$$

Employing (84)-(86), the covariance of $\hat{\rho}$ is

$$
\begin{aligned}
\operatorname{cov}(\hat{\boldsymbol{\rho}}) & =\mathbb{E}\left\{(\hat{\boldsymbol{\rho}}-\boldsymbol{\rho})(\hat{\boldsymbol{\rho}}-\boldsymbol{\rho})^{T}\right\} \\
& =\mathbf{C}^{-1} \operatorname{cov}(\hat{\mathbf{a}})\left(\mathbf{C}^{T}\right)^{-1} \\
& =\tilde{\lambda}_{1}^{-2} \sigma^{2} \mathbf{C}\left(\tilde{\mathbf{X}}^{T} \Psi(\mathbf{a}) \tilde{\mathbf{X}}\right)^{-1}\left(\mathbf{C}^{T}\right)^{-1} .
\end{aligned}
$$

As a result, the variances of $\hat{\mu}$ and $\hat{\alpha}$ are

$$
\operatorname{var}(\hat{\mu})=[\operatorname{cov}(\hat{\boldsymbol{\rho}})]_{1,1}
$$

and

$$
\operatorname{var}(\hat{\alpha})=[\operatorname{cov}(\hat{\boldsymbol{\rho}})]_{2,2} .
$$

Let $\hat{\boldsymbol{\chi}}=[\hat{\nu} \hat{\beta}]^{T}$. In a similar manner, the variances of $\hat{\nu}$ and $\hat{\beta}$ are determined as

$$
\operatorname{var}(\hat{\nu}) \approx[\operatorname{cov}(\hat{\chi})]_{1,1}
$$

and

$$
\operatorname{var}(\hat{\beta}) \approx[\operatorname{cov}(\hat{\chi})]_{2,2}
$$

where

$$
\operatorname{cov}(\hat{\boldsymbol{\chi}}) \approx \tilde{\lambda}_{1}^{-2} \sigma^{2} \mathbf{D}\left(\tilde{\mathbf{Y}}^{T} \mathbf{\Upsilon}(\mathbf{b}) \tilde{\mathbf{Y}}\right)^{-1}\left(\mathbf{D}^{T}\right)^{-1}
$$

and

$$
\mathbf{D}=\left[\begin{array}{cc}
-2 \beta \sin (\nu) & 2 \cos (\nu) \\
0 & -2 \beta
\end{array}\right]
$$

Though there are no closed-form expressions for (80)-(83) and (88)-(91), Section VI shows that their numerical values are equal to the corresponding CRLBs.

\section{Modifications for Partially DAMPED/UNDAMPED TONE}

For some applications, the tone is undamped in one [6] or even two dimensions [10]-[12]. In this section, we will show the required modifications for the PUMA approach when the cisoid/sinusoid is undamped in one dimension. The results for a purely undamped tone can be obtained in a similar manner.

\section{A. Complex Tone}

Assuming that $s_{m, n}$ of (2) is undamped in the second dimension, we have $\beta=1$ and $s_{m, n}=\gamma \alpha^{m} \exp (j(\mu m+\nu n))$. As a result, $\mathbf{h}$ of (6) becomes

$$
\mathbf{h}=\left[\begin{array}{llll}
\exp (j \nu) & \exp (j 2 \nu) & \cdots & \exp (j N \nu)
\end{array}\right]^{T} .
$$

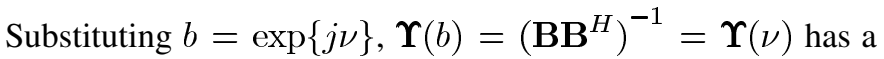
closed-form expression with elements [15]

$$
[\Upsilon(\nu)]_{m, n}=\frac{N \min (m, n)-m n}{N} \exp (j(n-m) \nu)
$$

and (31) can be simplified to

$$
\hat{\nu}=-\angle\left(\mathbf{y}_{1}^{H} \mathbf{\Upsilon}(\nu) \mathbf{y}_{2}\right)
$$


as the denominator is real and positive [15]. That is, estimation of $\nu$ is achieved by iterating between (95) and (96) while $\mu, \alpha$, and $\gamma$ are determined as in (22) and (35) with $\hat{\beta}=1$. The corresponding variance expressions also follow (80)-(83), except now $\beta=1$ in (82), that is

$$
\operatorname{var}(\hat{\nu}) \approx \frac{\sigma^{2}}{2 \tilde{\lambda}_{1}^{2} \tilde{\mathbf{y}}_{1}^{H} \boldsymbol{\Upsilon}(\nu) \tilde{\mathbf{y}}_{1}} .
$$

It is interesting to note that when $s_{m, n}$ is purely undamped, $|\mathbf{g}|=\sqrt{M},|\mathbf{h}|=\sqrt{N}$ and $\tilde{\lambda}_{1}=|\mathbf{g}||\mathbf{h}||\gamma|=\sqrt{M N}|\gamma|$. Recall (16) and let $\mathbf{h}_{1}=[\exp (j \nu) \exp (j 2 \nu) \cdots \exp (j(N-1) \nu)]^{T}$, we have

$$
\mathbf{h}_{1}^{*}=\sqrt{N} \exp \left(-j \varphi_{h}\right) \tilde{\mathbf{y}}_{1} .
$$

Employing (95) and (98) yields [15]

$$
\tilde{\mathbf{y}}_{1}^{H} \boldsymbol{\Upsilon}(\nu) \tilde{\mathbf{y}}_{1}=\frac{\mathbf{h}_{1}^{T}\left(\mathbf{B B}^{H}\right)^{-1} \mathbf{h}_{1}^{*}}{N}=\frac{N^{2}-1}{12} .
$$

As a result, $\operatorname{var}(\hat{\nu})$ has a closed-form of

$$
\operatorname{var}(\hat{\nu}) \approx \frac{6 \sigma^{2}}{\left(N^{2}-1\right) M N|\gamma|^{2}}
$$

which is the CRLB for frequency of a purely undamped cisoid [12].

\section{B. Real Tone}

Here, we consider $s_{m, n}=\gamma \alpha^{m} \cos (\mu m+\phi) \cos (\nu n+\theta)$ which corresponds to a real X-texture mode [6]. The vector $\mathbf{h}$ in (39) is modified as

$$
\mathbf{h}=\left[\begin{array}{llll}
\cos (\nu) & \cos (2 \nu) & \cdots & \cos (N \nu)
\end{array}\right]^{T} .
$$

The LP relationship in (41) is simplified to

$$
[\mathbf{h}]_{n}+[\mathbf{h}]_{n-2}=b_{1}[\mathbf{h}]_{n-1}, \quad n=3,4, \cdots, N
$$

where

$$
b_{1}=2 \cos (\nu)
$$

as $b_{2}=-1$. The vector equation for (101) to estimate $\nu$ becomes

$$
\mathbf{y}_{1} b_{1} \approx \mathbf{y}_{2}
$$

where $\mathbf{y}_{1}=\left[\left[\mathbf{v}_{1}\right]_{2}\left[\mathbf{v}_{1}\right]_{3} \cdots\left[\mathbf{v}_{1}\right]_{N-1}\right]^{T}$ and $\mathbf{y}_{2}=\left[\left[\mathbf{v}_{1}\right]_{1}+\right.$ $\left.\left[\mathbf{v}_{1}\right]_{3}\left[\mathbf{v}_{1}\right]_{2}+\left[\mathbf{v}_{1}\right]_{4} \cdots\left[\mathbf{v}_{1}\right]_{N-2}+\left[\mathbf{v}_{1}\right]_{N}\right]^{T}$. Hence, (63) is now modified to

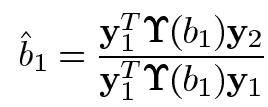

with $b_{2}=-1$ and $\hat{\nu}$ is computed as

$$
\hat{\nu}=\cos ^{-1}\left(\frac{\hat{b}_{1}}{2}\right) \text {. }
$$

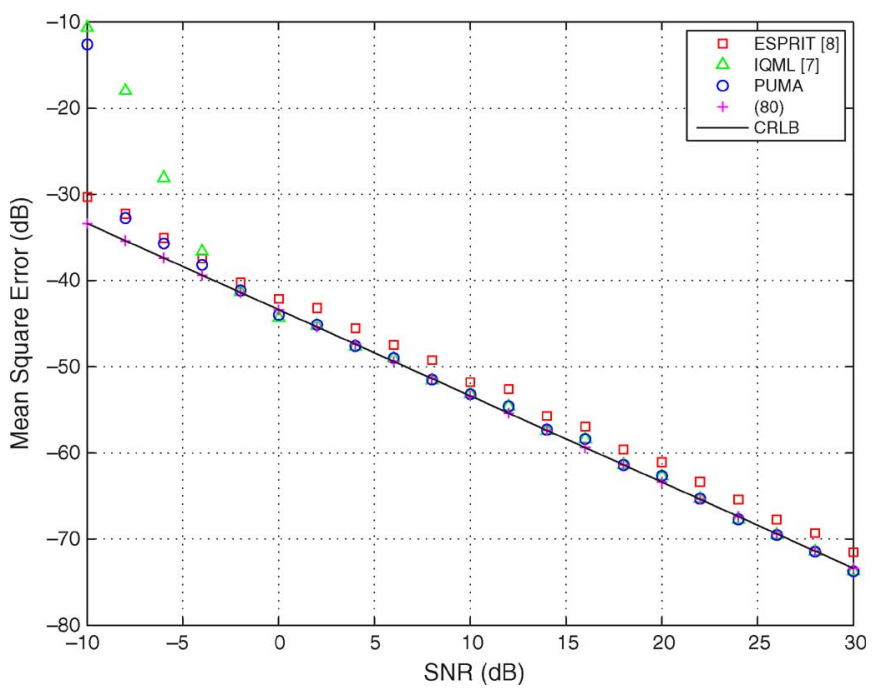

Fig. 1. Mean-square error of $\hat{\mu}$ versus SNR for damped cisoid.

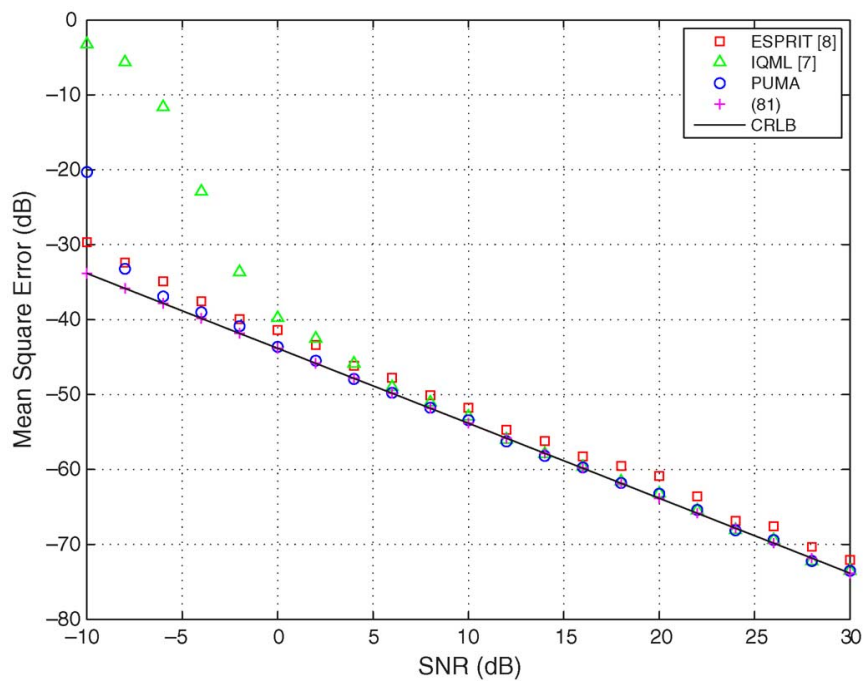

Fig. 2. Mean-square error of $\hat{\alpha}$ versus SNR for damped cisoid.

The estimates of $\alpha, \mu, \phi, \theta$ and $\gamma$ are calculated using (53), (54), (62), (68), and (72), respectively, with $\hat{\beta}=1$. Based on Section IV, the variance of $\hat{b}_{1}$ in (104) is evaluated as

$$
\operatorname{var}\left(\hat{b_{1}}\right) \approx \frac{\sigma^{2}}{\tilde{\lambda}_{1}^{2} \tilde{\mathbf{y}}_{1}^{T} \mathbf{\Upsilon}\left(b_{1}\right) \tilde{\mathbf{y}}_{1}} .
$$

Employing (105) and (106) yields [20]

$$
\operatorname{var}(\hat{\nu}) \approx \frac{\sigma^{2}}{4 \sin ^{2}(\nu) \tilde{\lambda}_{1}^{2} \tilde{\mathbf{y}}_{1}^{T} \mathbf{\Upsilon}\left(b_{1}\right) \tilde{\mathbf{y}}_{1}} .
$$

\section{Simulation Results}

Computer simulations have been carried out to evaluate the parameter estimation performance of the PUMA approach in the presence of white Gaussian noise. The stopping criterion of the PUMA algorithm is a fixed number of iterations. We use three 


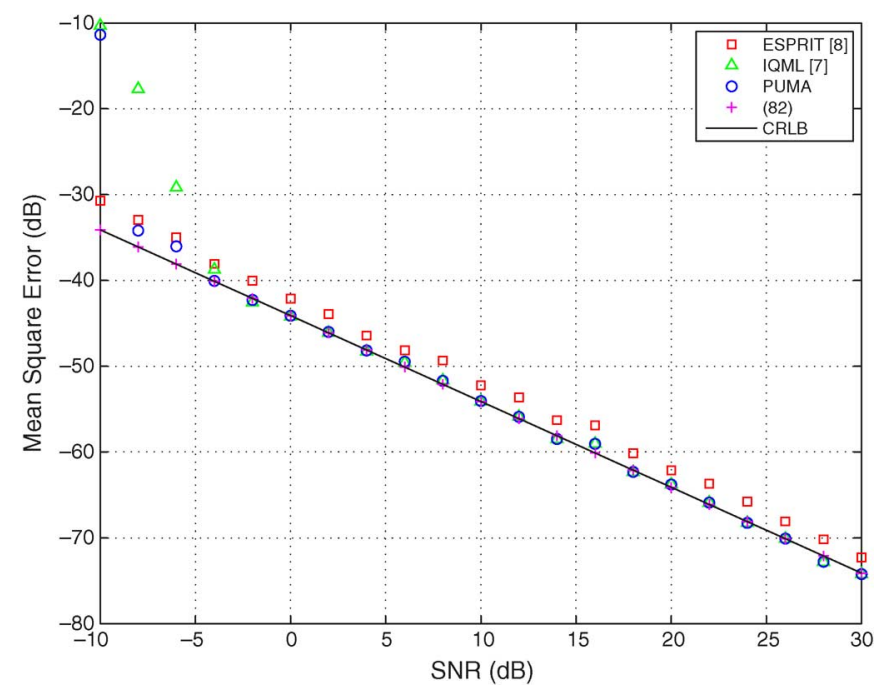

Fig. 3. Mean-square error of $\hat{\nu}$ versus SNR for damped cisoid.

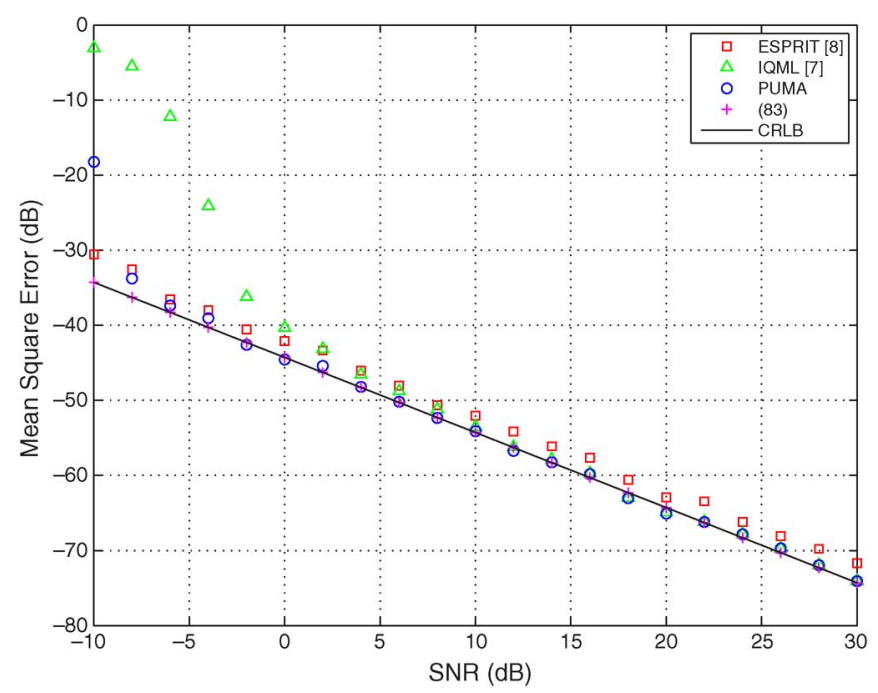

Fig. 4. Mean-square error of $\hat{\beta}$ versus SNR for damped cisoid.

iterations as no significant improvement is observed for more iterations. The mean-square error (MSE) is employed for the performance measure. For comparison, MSEs of the ESPRIT [8] and IQML [7] algorithms as well as CRLB are included for the complex model. While for the real signal case, [8] is replaced by [6], where partial forward-backward averaging is exploited. In the ESPRIT schemes [6], [8], a Hankel block-Hankel matrix of size around $M N / 4 \times(N / 2+1)(M / 2+1)$ is constructed from the $M \times N$ data matrix for parameter estimation. The signal power is defined as $\sum_{m=1}^{M} \sum_{n=1}^{N}\left|s_{m, n}\right|^{2} / M N$, and we scale the noise sequence to produce different SNR conditions. All results provided are averages of 200 independent runs using a computer with Pentium Dual Core 2-GHz processors and 1-GB RAM.

In the first test, we study the case of a damped complex tone, and the signal parameters are $\gamma=\exp (j), \alpha=0.95, \beta=0.98$, $\mu=0.1 \pi$, and $\nu=0.24 \pi$ with $M=N=20$. The estimation results for $\mu, \alpha, \nu, \beta$, and $\gamma$ versus SNR are plotted in Figs. 1-5, respectively. It is seen that the MSEs of the proposed scheme attain the corresponding CRLBs at SNR $\geq-2 \mathrm{~dB}$ in all five

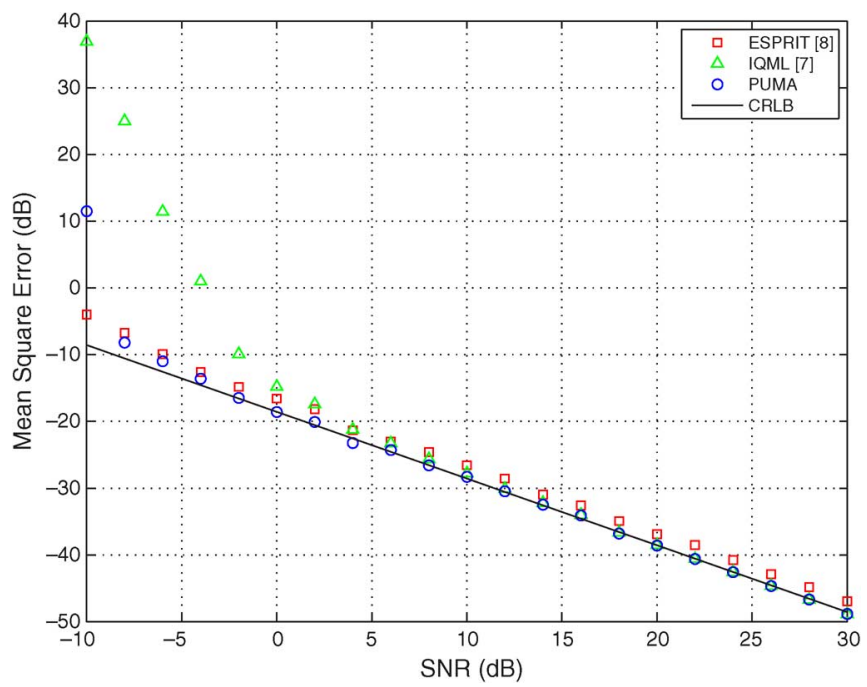

Fig. 5. Mean-square error of $\hat{\gamma}$ versus SNR for damped cisoid.

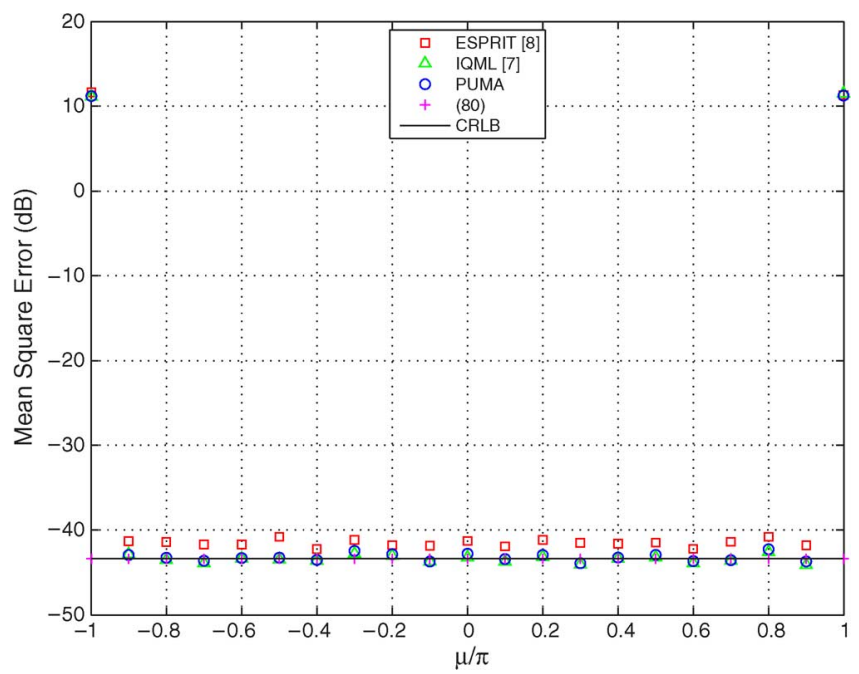

Fig. 6. Mean-square error of $\hat{\mu}$ versus $\mu$ for damped cisoid at SNR $=0 \mathrm{~dB}$.

figures. The theoretical variance expressions of (80)-(83) are also validated and they align with the optimum benchmark. As $\alpha<1$ and $\beta<1$, the MSE of the damping factor is less than that of the frequency, although the difference is not significant as both $\alpha$ and $\beta$ are close to unity. On the other hand, the IQML estimator can also provide optimum accuracy but it has larger threshold SNR than that of the PUMA approach, while the ESPRIT method is suboptimal in the whole SNR range. The average computation times of the ESPRIT, IQML and proposed algorithms for a single trial are measured as $6.37 \times 10^{-2} \mathrm{~s}$, $6.53 \mathrm{~s}$, and $2.28 \times 10^{-3} \mathrm{~s}$, respectively. Thus, the PUMA approach is more efficient than the ESPRIT and IQML estimators in terms of computational complexity and accuracy. In fact, its computationally attractiveness can be analytically deduced from the involved matrix size, which is $M \times N$. That is, the complexity of the proposed method is of $\mathcal{O}\left(M^{3}\right)$ due to the SVD and WLS operations. The involved matrix sizes in the ESPRIT and IQML algorithms are $M N / 4 \times(N / 2+1)(M / 2+1)$ and $(N(M-1)+(N-1) M) \times(N(M-1)+(N-1) M)$, respectively, indicating their complexities are both equal to $O\left(M^{6}\right)$. It 


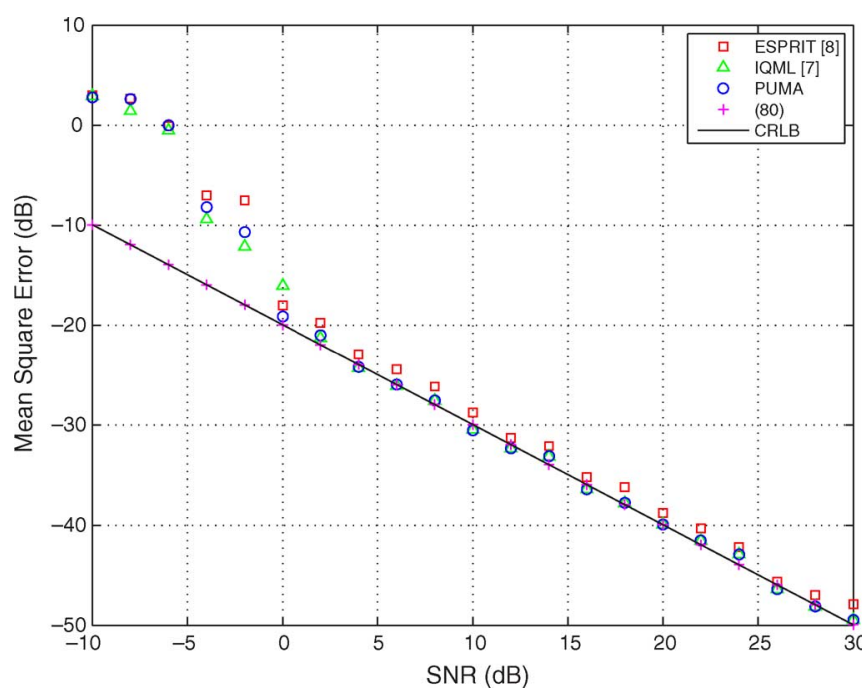

Fig. 7. Mean-square error of $\hat{\mu}$ versus SNR for damped cisoid at small data size.

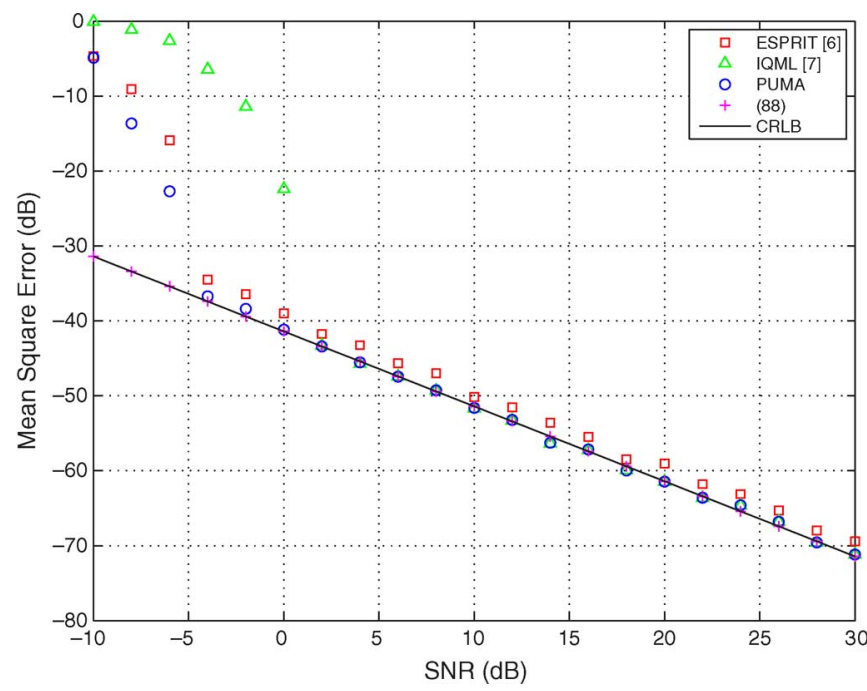

Fig. 8. Mean-square error of $\hat{\mu}$ versus SNR for X-texture mode.

is worth noting that the operations of the PUMA scheme can be further reduced by utilizing the power method to compute the principal singular vectors instead of performing the full SVD and by employing a fast algorithm for the sparse Toeplitz matrix inverse which may appear in the scientific computing literature.

Fig. 6 examines the estimation performance of $\hat{\mu}$ for $\mu \epsilon$ $(-\pi, \pi)$ at $\mathrm{SNR}=0 \mathrm{~dB}$, while the remaining parameters are identical to the previous experiment. The findings are similar to those of Fig. 1 although all estimators fail to achieve optimality when $\mu$ approaches $\pm \pi$. As the results of $\alpha, \nu, \beta$, and $\gamma$ are similar and indicate the uniform estimation performance, they are not provided here. Fig. 7 studies the performance for a smaller data size, namely, $M=N=5$, while the remaining parameters are identical to the first test. We observe that the estimation performance is similar to that of Fig. 1 except the threshold SNR is increased. The remaining parameters of interests give similar observations and their results are not included in this paper. As

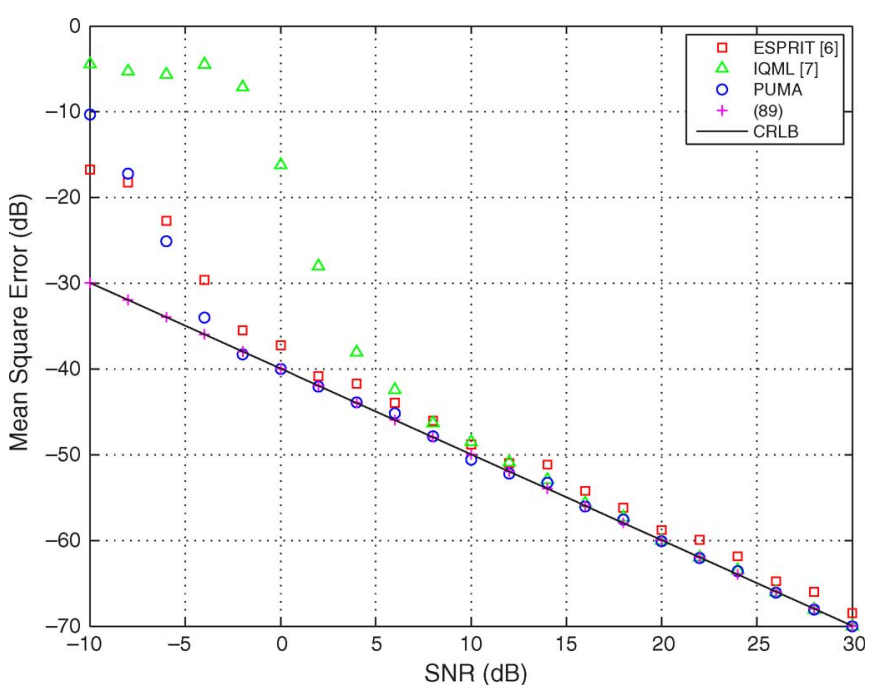

Fig. 9. Mean-square error of $\hat{\alpha}$ versus SNR for X-texture mode.

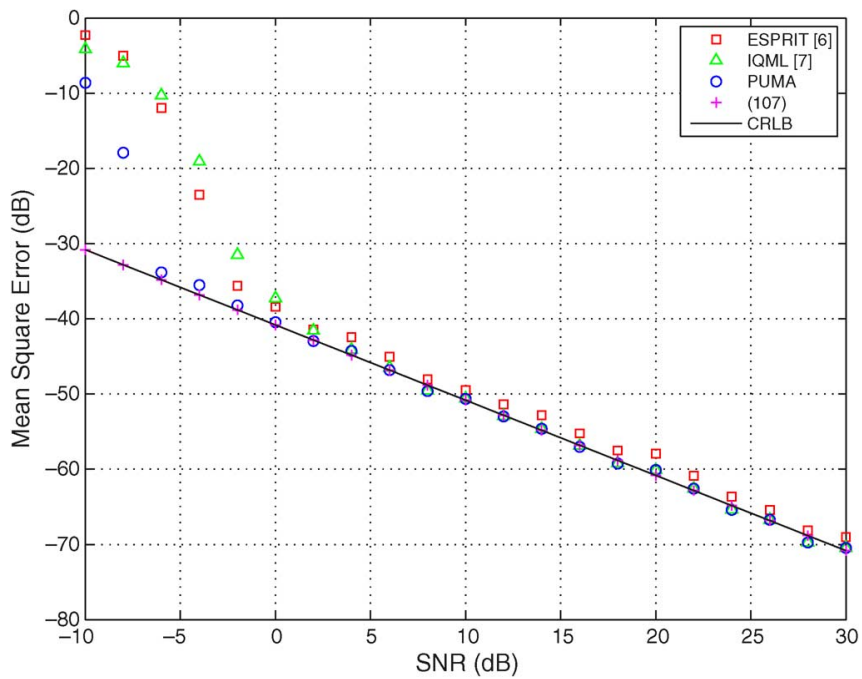

Fig. 10. Mean-square error of $\hat{\nu}$ versus SNR for X-texture mode.

a result, we can conclude that the PUMA algorithm can achieve CRLB at sufficiently large SNR and/or data size conditions.

Finally, we investigate the real tone and the signal parameters are $\gamma=1, \alpha=0.95, \mu=0.1 \pi, \nu=0.24 \pi, \phi=1$, and $\theta=2$ with $M=N=20$. That is, the real tone is undamped in one dimension which corresponds to the $\mathrm{X}$-texture mode [6]. The MSEs of $\hat{\mu}, \hat{\alpha}, \hat{\nu}, \hat{\phi}, \hat{\theta}$, and $\hat{\gamma}$ are shown in Figs. 8-13, respectively. Again, we see that the PUMA algorithm is superior to the ESPRIT and IQML methods as in Figs. 1-5 and its performance is able to achieve the CRLB for sufficiently high SNRs. In addition, the average computation times of the ESPRIT, IQML and proposed estimators for a single trial are measured as are $4.11 \times 10^{-2} \mathrm{~s}, 2.85 \mathrm{~s}$ and $2.65 \times 10^{-3} \mathrm{~s}$, respectively.

\section{CONCLUSION}

We have devised an efficient parameter estimation approach for a two-dimensional (2-D) single damped real/complex tone 


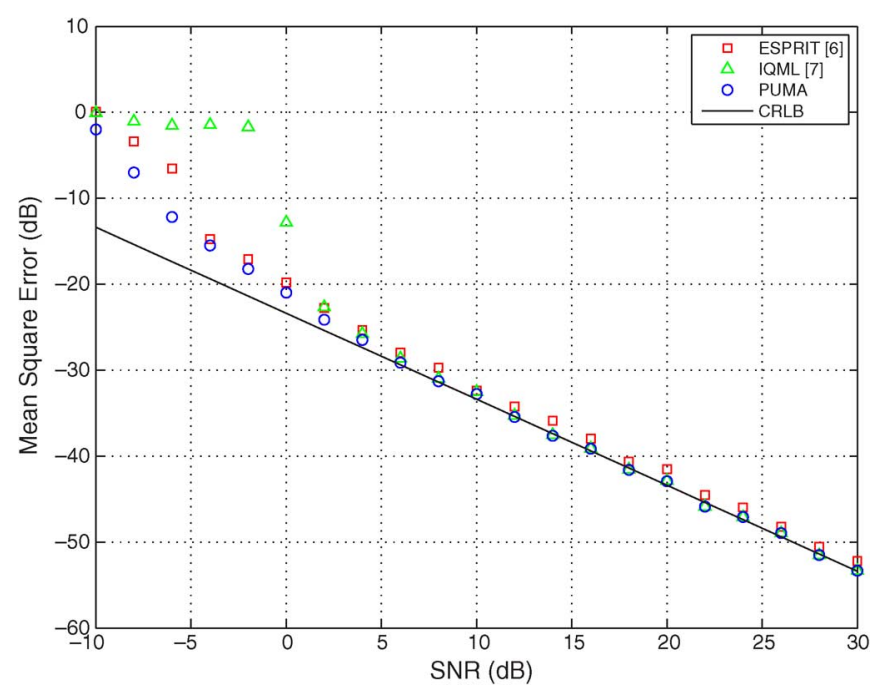

Fig. 11. Mean-square error of $\hat{\phi}$ versus SNR for X-texture mode.

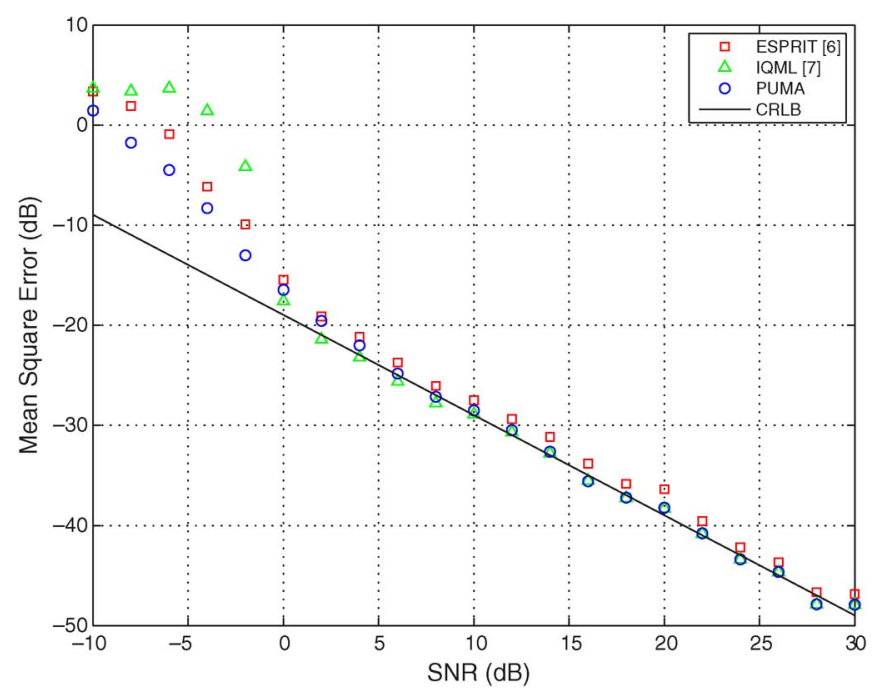

Fig. 12. Mean-square error of $\hat{\theta}$ versus SNR for X-texture mode.

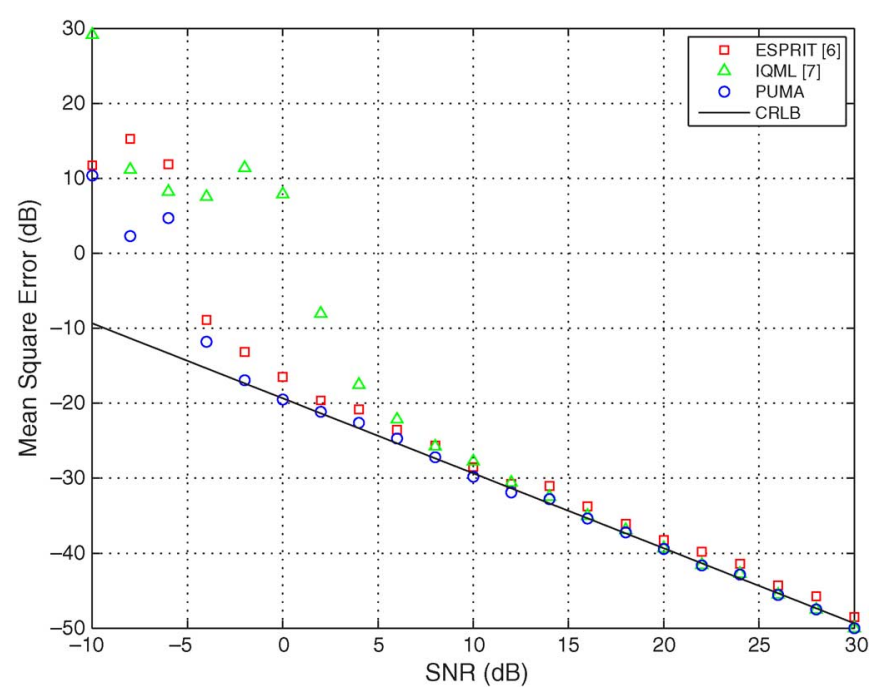

Fig. 13. Mean-square error of $\hat{\gamma}$ versus SNR for X-texture mode. in additive white Gaussian noise and we refer it to as principal-singular-vector utilization for modal analysis (PUMA). The key ideas are to make use of the rank-one property of the 2-D noise-free data matrix and find the damping factor as well as frequency parameters for each dimension from the principal left and right singular vectors in a separable manner according to an iterative weighted least squares procedure. Modifications for a tone which is undamped in at least one dimension are included. Mean and variance expressions for the damping factor and frequency parameters are also produced and verified via computer simulations, which illustrate that they are approximately unbiased and their performance achieves Cramér-Rao lower bound at sufficiently large signal-to-noise ratio and/or data size conditions. Furthermore, it is shown that the PUMA approach outperforms the iterative quadratic maximum likelihood [7] and subspace based algorithms [6], [8] in terms of computational complexity and accuracy. Considering 2-D single-tone parameter estimation as a starting point, we will extend our study to the multidimensional [21], [22] and multiple-tone scenarios as our future works.

\section{REFERENCES}

[1] J. Li, P. Stoica, and D. Zheng, "An efficient algorithm for two-dimensional frequency estimation," Multidimension. Syst. Signal Process., vol. 7, pp. 151-178, 1996.

[2] J. W. Odendaal, E. Barnard, and W. I. Pistorius, "Two-dimensional superresolution radar imaging using the MUSIC algorithm," IEEE Trans. Antennas Propag., vol. 42, no. 10, pp. 1386-1391, Oct. 1994.

[3] X. Xie and R. J. Evans, "Frequency-wavenumber tracking using hidden Markov models," IEEE Trans. Signal Process., vol. 41, no. 3, pp. 1391-1394, Mar. 1993.

[4] Y. Li, J. Razavilar, and K. J. R. Liu, "A high-resolution technique for multidimensional NMR spectroscopy," IEEE Trans. Biomed. Eng., vol. 45, no. 1, pp. 78-86, Jan. 1998.

[5] A.-J. van der Veen, M. C. Vanderveen, and A. Paulraj, "Joint angle and delay estimation using shift-invariance techniques," IEEE Trans. Signal Process., vol. 46, no. 2, pp. 405-418, Feb. 1998.

[6] J. Axmon, M. Hansson, and L. Sornmo, "Partial forward-backward averaging for enhanced frequency estimation of real X-texture modes," IEEE Trans. Signal Process., vol. 53, no. 7, pp. 2550-2562, Jul. 2005.

[7] M. P. Clark and L. L. Scharf, "Two-dimensional modal analysis based on maximum likelihood," IEEE Trans. Signal Process., vol. 42, no. 6, pp. 1443-1452, Jun. 1994.

[8] S. Rouquette and M. Najim, "Estimation of frequencies and damping factors by two-dimensional ESPRIT type methods," IEEE Trans. Signal Process., vol. 49, no. 1, pp. 237-245, Jan. 2001.

[9] Y. Hua and T. N. Sarkar, "Matrix pencil method for estimating parameters of exponentially damped/undamped sinusoids in noise," IEEE Trans. Acoust., Speech, Signal Process., vol. 38, no. 5, pp. 814-824, May 1990.

[10] Y. Hua, "Estimating two-dimensional frequencies by matrix enhancement and matrix pencil," IEEE Trans. Signal Process., vol. 40, no. 9, pp. 2267-2280, Sep. 1992.

[11] S. Kay and R. Nekovei, "An efficient two-dimensional frequency estimator," IEEE Trans. Acoust., Speech, Signal Process., vol. 38, no. 10, pp. 1807-1809, Oct. 1990.

[12] H. C. So and K. W. Chan, "Approximate maximum likelihood algorithms for two-dimensional frequency estimation of a complex sinusoid," IEEE Trans. Signal Process., vol. 54, no. 8, pp. 3231-3237, Aug. 2006.

[13] R. Kumaresan, L. L. Scharf, and A. K. Shaw, "An algorithm for polezero modeling and spectral analysis," IEEE Trans. Acoust., Speech, Signal Process., pp. 637-640, Jun. 1986.

[14] Y. Bresler and A. Macovski, "Exact maximum likelihood parameter estimation of superimposed exponential signals in noise," IEEE Trans. Acoust., Speech, Signal Process., vol. 34, no. 5, pp. 1081-1088, Oct. 1986.

[15] H. C. So and K. W. Chan, "A generalized weighted linear predictor frequency estimation approach for a complex sinusoid," IEEE Trans. Signal Process., vol. 54, no. 4, pp. 1304-1315, Apr. 2006. 
[16] T. Soderstrom and P. Stoica, System Identification. Englewood Cliffs, NJ: Prentice-Hall, 1989.

[17] S. M. Kay, Fundamentals of Statistical Signal Processing: Estimation Theory. Englewood Cliffs, NJ: Prentice-Hall, 1993.

[18] J. Liu, X. Liu, and X. Ma, "First-order perturbation analysis of singular vectors in singular value decomposition," IEEE Trans. Signal Process., vol. 56, no. 7, pp. 3044-3049, Jul. 2008.

[19] Y.-X. Yao and S. M. Pandit, "Variance of least squares estimators for a damped sinusoidal process," IEEE Trans. Signal Process., vol. 42, no. 11, pp. 3016-3025, Nov. 1994.

[20] H. C. So and K. W. Chan, "Reformulation of Pisarenko harmonic decomposition method for single-tone frequency estimation," IEEE Trans. Signal Process., vol. 52, no. 4, pp. 1128-1135, Apr. 2004.

[21] J. Liu and X. Liu, "An eigenvector-based approach for multidimensional frequency estimation with improved identifiability," IEEE Trans. Signal Process., vol. 54, no. 12, pp. 4543-4556, Dec. 2006.

[22] J. Liu, X. Liu, and X. Ma, "Multidimensional frequency estimation with finite snapshots in the presence of identical frequencies," IEEE Trans. Signal Process., vol. 55, no. 11, pp. 5179-5194, Nov. 2007.

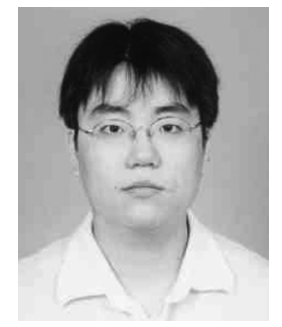

Frankie K. W. Chan received the B.Eng. degree in computer engineering and the Ph.D. degree from the City University of Hong Kong in 2002 and 2008, respectively.

He is currently a Research Fellow in the same university. His research interests include parameter estimation, optimization and distributed processing, with particular attention to frequency estimation and node localization in wireless sensor network.

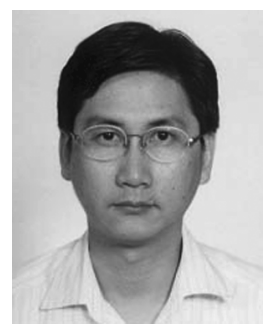

W. H. Lau received the B.Sc. and Ph.D. degrees in electrical and electronic engineering from the University of Portsmouth in 1985 and 1989, respectively.

In 1990, he joined the City University of Hong Kong, where he is currently an Associate Professor in the Department of Electronic Engineering. His current research interests are in the area of digital signal processing, digital audio engineering, and PWM spectrum analysis.

Dr. Lau was the recipient of the IEEE Third MilIEEE Hong Kong Section in 2005. lennium Medal. He served as the Chairman of the

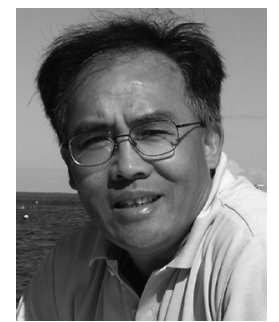

Cheung-Fat Chan was born in Hong Kong in 1957. $\mathrm{He}$ received the B.Sc., M.Sc., and Ph.D. degrees in electronic engineering, all from University of Essex, U.K., in 1983, 1984, and 1987, respectively.

In 1988, he joined the City University of Hong Kong, where he is currently an Associate Professor in the Department of Electronic Engineering, City University of Hong Kong. In 1994, he co-founded TeleEye Ltd., an associated company of City University of Hong Kong, which develops innovative audio and video compression techniques for industrial electronic products. The company was listed in Hong Kong Stock Exchange in 2001. He is now a non-Executive Director of the listed company. His main research interests are in speech and audio coding, processing, and fast algorithms for noise suppression and enhancement.

Dr. Chan is the past Chairman of the IEEE signal processing chapter in Hong Kong (1997-1998). 